\title{
Sufficient Conditions for Univalence of an Integral Operator
}

\author{
Georgia Irina Oros, ${ }^{1}$ Gheorghe Oros, ${ }^{1}$ and Daniel Breaz ${ }^{2}$ \\ ${ }^{1}$ Department of Mathematics, University of Oradea, str. Universitatii nr. 1, 410087 Oradea, Romania \\ ${ }^{2}$ Department of Mathematics and Computer Science, 1 Decembrie 1918 University of Alba Iulia, \\ str. N. Iorga, no. 11-13, 510009 Alba Iulia, Romania
}

Correspondence should be addressed to Daniel Breaz,dbreaz@uab.ro

Received 27 February 2008; Accepted 24 May 2008

Recommended by Ulrich Abel

In this paper we have introduced an integral general operator. For this general operator which is a generalization of more known integral operators we have demonstrated some univalence properties.

Copyright (c) 2008 Georgia Irina Oros et al. This is an open access article distributed under the Creative Commons Attribution License, which permits unrestricted use, distribution, and reproduction in any medium, provided the original work is properly cited.

\section{Introduction and preliminaries}

Let $U$ be the unit disk of the complex plane:

$$
U=\{z \in \mathbb{C}:|z|<1\} .
$$

Let $\mathscr{\ell}(U)$ be the space of holomorphic functions in $U$,

$$
A_{n}=\left\{f \in \mathscr{H}(U), f(z)=z+a_{n+1} z^{n+1}+\cdots, z \in U\right\}
$$

with $A_{1}=A$, and

$$
S=\{f \in A: f \text { is univalent in } U\} .
$$

Lemma 1.1 (see [1]). If the function $f$ is regular in the unit disc $U$,

$$
\begin{gathered}
f(z)=z+a_{2} z^{2}+\cdots, \\
\left(1-|z|^{2}\right)\left|\frac{z f^{\prime \prime}(z)}{f^{\prime}(z)}\right| \leq 1 \quad \forall z \in U,
\end{gathered}
$$

then the function $f$ is univalent in $U$. 
Definition 1.2 (St. Ruscheweyh [2]). For $f \in A, n \in \mathbb{N} \cup\{0\}$, let $R^{n}$ be the operator defined by $R^{n}: A \rightarrow A$,

$$
\begin{aligned}
R^{0} f(z) & =f(z), \\
R^{1} f(z) & =z f^{\prime}(z) \\
\vdots & \\
(n+1) R^{n+1} f(z) & =z\left[R^{n} f(z)\right]^{\prime}+n R^{n} f(z), \quad z \in U .
\end{aligned}
$$

Remark 1.3. If $f \in A$

$$
f(z)=z+\sum_{j=2}^{\infty} a_{j} z^{j}
$$

then

$$
R^{n} f(z)=z+\sum_{j=1}^{\infty} C_{n+j-1}^{n} a_{j} z^{j}, \quad z \in U
$$

with

$$
R^{n} f(0)=0, \quad\left[R^{n} f(0)\right]^{\prime}=1
$$

Lemma 1.4 ([3, Schwarz's lemma], [4, Lemma 4.26, page 103]). If the analytic function $f(z)$ is regular in $U$ with $f(0)=0$ and $|f(z)|<1$ for all $z \in U$, then

$$
|f(z)| \leq|z|, \quad \forall z \in U,
$$

and $\left|f^{\prime}(0)\right| \leq 1$.

The equality holds if and only if $f(z)=c z, z \in U,|c|=1$.

\section{Main results}

By using the Ruscheweyh differential operator given by Definition 1.2, we introduce the following integral operator.

Definition 2.1. Let $n, m \in \mathbb{N} \cup\{0\}, i \in\{1,2,3, \ldots, m\}, \alpha_{i} \in \mathbb{C}$. Define the integral operator $I\left(f_{1}, f_{2}, \ldots, f_{m}\right): A^{m} \rightarrow A$,

$$
I\left(f_{1}, f_{2}, \ldots, f_{m}\right)(z)=\int_{0}^{z}\left[\frac{R^{n} f_{1}(t)}{t}\right]^{\alpha_{1}} \cdots\left[\frac{R^{n} f_{m}(t)}{t}\right]^{\alpha_{m}} d t, \quad z \in U,
$$

where $f_{i}(z) \in A$ and $R^{n}$ is the Ruscheweyh differential operator.

Remark 2.2. (i) For $n=0, m=1, \alpha_{1}=1, \alpha_{2}=\alpha_{3}=\cdots=\alpha_{m}=0$,

$$
R^{0} f(z)=f(z) \in A
$$


we obtain Alexander integral operator introduced in 1915 in [5]:

$$
I(z)=\int_{0}^{z} \frac{f(t)}{t} d t, \quad z \in U
$$

(ii) For $n=0, m=1, \alpha_{1}=\alpha \in[0,1], \alpha_{2}=\alpha_{3}=\cdots=\alpha_{m}=0, R^{0} f(z)=f(z) \in S$, and we obtain the integral operator

$$
I_{\alpha}(z)=\int_{0}^{z}\left[\frac{f(t)}{t}\right]^{\alpha} d t
$$

studied in [6].

(iii) For $n=1, m=1, \alpha_{1}=\gamma \in \mathbb{C},|\gamma| \leq 1 / 4, \alpha_{2}=\cdots=\alpha_{m}=0, R^{1} f(z)=z f^{\prime}(z) \in S$, we obtain the integral operator

$$
F_{\gamma}(z)=\int_{0}^{z}\left[f^{\prime}(t)\right]^{\gamma} d t
$$

studied in $[7,8]$.

(iv) For $n=0, m \in \mathbb{N} \cup\{0\}, \alpha_{i} \in \mathbb{C}, i \in\{1,2, \ldots, m\}, R^{0} f(z)=f(z) \in S$, and we obtain the integral operator

$$
F(z)=\int_{0}^{z}\left[\frac{f_{1}(t)}{t}\right]^{\alpha_{1}} \cdots\left[\frac{f_{m}(t)}{t}\right]^{\alpha_{m}} d t
$$

studied in [9].

(v) For $n, m \in \mathbb{N} \cup\{0\}, i \in\{1,2, \ldots, m\}, \alpha_{i}>0$, we obtain the integral operator $F_{m}$ : $A^{m} \rightarrow A$,

$$
F_{m}\left(f_{1}, f_{2}, \ldots, f_{m}\right)(z)=\int_{0}^{z}\left[\frac{R^{n} f_{1}(t)}{t}\right]^{\alpha_{1}} \cdots\left[\frac{R^{n} f_{m}(t)}{t}\right]^{\alpha_{m}} d t
$$

studied in [10].

(vi) For $n=0, m=1, \alpha_{1}=\gamma, \alpha_{2}=\cdots=\alpha_{m}=0, R^{0} f(z)=f(z)$, and we obtain the integral operator

$$
F_{\gamma}(z)=\int_{0}^{z}\left[\frac{f(t)}{t}\right]^{\gamma} d t
$$

studied in $[11,12]$.

Theorem 2.3. Let $n, m \in \mathbb{N} \cup\{0\}, i \in\{1,2, \ldots, m\}, \alpha_{i} \in \mathbb{C}, f_{i} \in A$. If

$$
\left|\frac{z\left(R^{n} f_{i}(z)\right)^{\prime}}{R^{n} f_{i}(z)}-1\right| \leq 1, \quad\left|\alpha_{1}\right|+\left|\alpha_{2}\right|+\cdots+\left|\alpha_{m}\right| \leq 1, \quad z \in U,
$$

then $I\left(f_{1}, f_{2}, \ldots, f_{m}\right)(z)$ given by $(2.1)$ is univalent. 
Proof. Since $f_{i} \in A, i \in\{1,2, \ldots, m\}$, from Remark 1.3 we have

$$
\begin{gathered}
\frac{R^{n} f_{i}(z)}{z}=\frac{z+\sum_{j=2}^{\infty} C_{n+j-1}^{n} a_{j, i} z^{j}}{z}=1+\sum_{j=2}^{\infty} C_{n+j-1}^{n} a_{j, i} z^{j-1}, \\
\frac{R^{n} f_{i}(z)}{z} \neq 0, \quad z \in U .
\end{gathered}
$$

For $z=0$, we have

$$
\left[\frac{R^{n} f_{1}(z)}{z}\right]^{\alpha_{1}} \cdots\left[\frac{R^{n} f_{m}(z)}{z}\right]^{\alpha_{m}}=1
$$

By differentiating (2.1), we obtain

$$
\begin{gathered}
I^{\prime}\left(f_{1}, f_{2}, \ldots, f_{m}\right)(z)=\left[\frac{R^{n} f_{1}(z)}{z}\right]^{\alpha_{1}} \cdots\left[\frac{R^{n} f_{m}(z)}{z}\right]^{\alpha_{m}}, \quad z \in U, \\
I^{\prime}\left(f_{1}, f_{2}, \ldots, f_{m}\right)(0)=1 .
\end{gathered}
$$

Using (2.12), we obtain

$\log I^{\prime}\left(f_{1}, f_{2}, \ldots, f_{m}\right)(z)=\alpha_{1}\left[\log R^{n} f_{1}(z)-\log z\right]+\cdots+\alpha_{m}\left[\log R^{n} f_{m}(z)-\log z\right], \quad z \in U$

By differentiating (2.13), we have

$$
\frac{I^{\prime \prime}\left(f_{1}, f_{2}, \ldots, f_{m}\right)(z)}{I^{\prime}\left(f_{1}, f_{2}, \ldots, f_{m}\right)(z)}=\alpha_{1}\left[\frac{\left(R^{n} f_{1}(z)\right)^{\prime}}{R^{n} f_{1}(z)}-\frac{1}{z}\right]+\cdots+\alpha_{m}\left[\frac{\left(R^{n} f_{m}(z)\right)^{\prime}}{R^{n} f_{m}(z)}-\frac{1}{z}\right], \quad z \in U
$$

and after a short calculus we obtain

$$
\frac{z I^{\prime \prime}\left(f_{1}, f_{2}, \ldots, f_{m}\right)(z)}{I^{\prime}\left(f_{1}, f_{2}, \ldots, f_{m}\right)(z)}=\left|\alpha_{1}\right|\left[\frac{z\left(R^{n} f_{1}(z)\right)^{\prime}}{R^{n} f_{1}(z)}-1\right]+\cdots+\left|\alpha_{m}\right|\left[\frac{z\left(R^{n} f_{m}(z)\right)^{\prime}}{R^{n} f_{m}(z)}-1\right], \quad z \in U .
$$

We multiply the modulus of (2.15) by $\left(1-|z|^{2}\right)$ and we obtain

$$
\begin{aligned}
\left(1-|z|^{2}\right)\left|\frac{z I^{\prime \prime}\left(f_{1}, f_{2}, \ldots, f_{m}\right)(z)}{I^{\prime}\left(f_{1}, f_{2}, \ldots, f_{m}\right)(z)}\right| \\
\quad=\left(1-|z|^{2}\right)\left|\alpha_{1}\left[\frac{z\left(R^{n} f_{1}(z)\right)^{\prime}}{R^{n} f_{1}(z)}-1\right]+\cdots+\alpha_{m}\left[\frac{z\left(R^{n} f_{m}(z)\right)^{\prime}}{R^{n} f_{m}(z)}-1\right]\right| \\
\quad \leq\left(1-|z|^{2}\right)\left[\left|\alpha_{1}\right|\left|\frac{z\left(R^{n} f_{1}(z)\right)}{R^{n} f_{1}(z)}-1\right|+\cdots+\left|\alpha_{m}\right|\left|\frac{z\left(R^{n} f_{m}(z)\right)}{R^{n} f_{m}(z)}-1\right|\right] \\
\quad \leq\left[\left|\alpha_{1}\right|+\cdots+\left|\alpha_{m}\right|\right]\left(1-\left|z^{2}\right|\right) \leq\left|\alpha_{1}\right|+\cdots+\left|\alpha_{m}\right| \leq 1 .
\end{aligned}
$$

From Lemma A, we have $I\left(f_{1}, f_{2}, \ldots, f_{m}\right)(z) \in S$. 
Remark 2.4. (i) For $n=0, R^{n} f_{i}(z)=f_{i}(z) \in S$, we obtain Theorem 2.3 from [9].

(ii) For $\alpha_{i} \in \mathbb{R}, \alpha_{i}>0$, Theorem 2.3 can be rewritten as follows.

Corollary 2.5. Let $n, m \in \mathbb{N} \cup\{0\}, i \in\{1,2, \ldots, m\}, \alpha_{i}>0$ with $\alpha_{1}+\alpha_{2}+\cdots+\alpha_{m} \leq 1$. If $f_{i} \in A$ satisfy

$$
\left|\frac{z\left(R^{n} f_{i}(z)\right)^{\prime}}{R^{n} f_{i}(z)}-1\right| \leq 1, \quad z \in U
$$

then the integral operator given by (2.1) is univalent.

Theorem 2.6. Let $n, m \in \mathbb{N} \cup\{0\}, i \in\{1,2, \ldots, m\}, \alpha_{i} \in \mathbb{C}$. If $f_{i} \in A$ satisfy

(i) $\left|\alpha_{1}\right|+\cdots+\left|\alpha_{m}\right| \leq 1 / 3$,

(ii) $\left|R^{n} f_{i}(z)\right| \leq 1$,

(iii) $\left|z^{2}\left(R^{n} f_{i}(z)\right)^{\prime} /\left(R_{i}^{n} f_{i}(z)\right)^{2}-1\right|<1$

for all $z \in U$, then the integral operator given by (2.1) is univalent.

Proof. Using (2.14), we obtain

$$
\left|\frac{z\left[I\left(f_{1}, \ldots, f_{m}\right)(z)\right]}{\left[I\left(f_{1}, \ldots, f_{m}\right)(z)\right]^{\prime}}\right|^{\prime \prime}=\left|\alpha_{1}\right|\left|\frac{z\left(R^{n} f_{1}(z)\right)^{\prime}}{R^{n} f_{1}(z)}-1\right|+\ldots+\left|\alpha_{m}\right|\left|\frac{z\left(R^{n} f_{m}(z)\right)^{\prime}}{R^{n} f_{m}(z)}-1\right|
$$

We multiply (2.18) by $\left(1-|z|^{2}\right)$, use Schwarz's lemma, and obtain

$$
\begin{aligned}
\left(1-|z|^{2}\right)\left|\frac{z T^{\prime \prime}(z)}{T^{\prime}(z)}\right| & \left(1-|z|^{2}\right)\left|\alpha_{1}\right|\left|\frac{z\left(R^{n} f_{1}(z)\right)}{R^{n} f_{1}(z)}-1\right|+\cdots+\left(1-|z|^{2}\right)\left|\alpha_{m}\right|\left|\frac{z\left(R^{n} f_{m}(z)\right)^{\prime}}{R^{n} f_{1}(z)}-1\right| \\
= & \left(1-|z|^{2}\right)\left|\alpha_{1}\right|\left|\frac{z\left(R^{n} f_{1}(z)\right)^{\prime}}{R^{n} f_{1}(z)}\right|+\left(1-|z|^{2}\right)\left|\alpha_{1}\right|+\cdots+\left(1-|z|^{2}\right)\left|\alpha_{m}\right|\left|\frac{z\left(R^{n} f_{m}(z)\right)^{\prime}}{R^{n} f_{1}(z)}\right| \\
& +\left(1-|z|^{2}\right)\left|\alpha_{m}\right| \\
= & \left(1-|z|^{2}\right)\left|\alpha_{1}\right|\left[\left|\frac{z\left(R^{n} f_{1}(z)\right)^{\prime}}{R^{n} f_{1}(z)}\right|+\cdots+\left|\frac{z\left(R^{n} f_{m}(z)\right)^{\prime}}{R^{n} f_{1}(z)}\right|\right]+\left(1-|z|^{2}\right)\left[\left|\alpha_{1}\right|+\cdots+\left|\alpha_{m}\right|\right] \\
= & \left(1-|z|^{2}\right)\left[\left|\alpha_{1}\right|\left|\frac{z^{2}\left(R^{n} f_{1}(z)\right)^{\prime}}{\left(R^{n} f_{1}(z)\right)^{2}}\right| \frac{\left|R^{n} f_{1}\right|}{|z|}+\cdots+\left|\alpha_{m}\right|\left|\frac{z^{2}\left(R^{n} f_{m}(z)\right)^{\prime}}{\left(R^{n} f_{m}(z)\right)^{2}}\right| \frac{\left|R^{n} f_{m}\right|}{|z|}\right] \\
& +\left(1-|z|^{2}\right)\left[\left|\alpha_{1}\right|+\cdots+\left|\alpha_{m}\right|\right]
\end{aligned}
$$




$$
\begin{aligned}
\leq & \left(1-|z|^{2}\right)\left[\left|\alpha_{1}\right| \frac{z^{2}\left(R^{n} f_{1}(z)\right)^{\prime}}{\left(R^{n} f_{1}(z)\right)^{2}}|+\cdots+| \alpha_{m}|| \frac{z^{2}\left(R^{n} f_{m}(z)\right)^{\prime}}{\left(R^{n} f_{m}(z)\right)^{2}} \mid\right]+\left(1-|z|^{2}\right)\left[\left|\alpha_{1}\right|+\cdots+\left|\alpha_{m}\right|\right] \\
= & \left(1-|z|^{2}\right)\left[\left|\alpha_{1}\right| \frac{z^{2}\left(R^{n} f_{1}(z)\right)^{\prime}}{\left(R^{n} f_{1}(z)\right)^{2}}|-| \alpha_{1}|+| \alpha_{1} \mid\right] \\
& +\cdots+\left(1-|z|^{2}\right)\left[\left|\alpha_{m}\right|\left|\frac{z^{2}\left(R^{n} f_{m}(z)\right)^{\prime}}{\left(R^{n} f_{m}(z)\right)^{2}}\right|-\left|\alpha_{m}\right|+\left|\alpha_{m}\right|\right]+\left(1-|z|^{2}\right)\left[\left|\alpha_{1}\right|+\cdots+\left|\alpha_{m}\right|\right] \\
= & \left(1-|z|^{2}\right)\left[\left|\alpha_{1}\right|\left|\frac{z^{2}\left(R^{n} f_{1}(z)\right)^{\prime}}{\left(R^{n} f_{1}(z)\right)^{2}}-1\right|+\cdots+\left|\alpha_{m}\right|\left|\frac{z^{2}\left(R^{n} f_{m}(z)\right)^{\prime}}{\left(R^{n} f_{m}(z)\right)^{2}}-1\right|\right] \\
& +\left(1-|z|^{2}\right)\left(\left|\alpha_{1}\right|+\cdots+\left|\alpha_{m}\right|\right)+\left(1-|z|^{2}\right)\left(\left|\alpha_{1}\right|+\cdots+\left|\alpha_{m}\right|\right) \\
\leq & \left(1-|z|^{2}\right)\left(\left|\alpha_{1}\right|+\left|\alpha_{1}\right|+\cdots+\left|\alpha_{m}\right|\right)+2\left(1-|z|^{2}\right)\left(\left|\alpha_{1}\right|+\cdots+\left|\alpha_{m}\right|\right) \\
= & 3\left(1-|z|^{2}\right)\left(\left|\alpha_{1}\right|+\cdots+\left|\alpha_{m}\right|\right) \\
\leq & 3\left(\left|\alpha_{1}\right|+\cdots+\left|\alpha_{m}\right|\right) .
\end{aligned}
$$

From (2.19) and condition (i), we have

$$
\left(1-|z|^{2}\right)\left|\frac{z F^{\prime \prime}(z)}{F^{\prime}(z)}\right| \leq 1
$$

for all $z \in U$.

By Lemma A, it follows that the integral operator $I\left(f_{1}, f_{2}, \ldots, f_{m}\right)(z)$ is univalent.

Remark 2.7. For $n=0, m=1, \alpha_{1}=\alpha \in \mathbb{C},|\alpha| \leq 1 / 3, \alpha_{2}=\cdots=\alpha_{m}=0$, the result was obtained in [11, Theorem 1].

For $\alpha_{i} \in \mathbb{R}, \alpha_{i}>0$, Theorem 2.6 can be rewritten as follows.

Corollary 2.8. Let $n, m \in \mathbb{N} \cup\{0\}, i \in\{1,2, \ldots, m\}, \alpha_{i}>0$. If $f_{i} \in$ A satisfy

(i) $\alpha_{1}+\alpha_{2}+\cdots+\alpha_{n} \leq 1 / 3$,

(ii) $\left|R^{n} f_{i}(z)\right| \leq 1$,

(iii) $\left|z^{2}\left(R^{n} f_{i}(z)\right)^{\prime} /\left(R^{n} f_{i}(z)\right)^{2}-1\right|<1$

for all $z \in U$, then the integral operator given by (2.1) is univalent.

\section{References}

[1] J. Becker, "Löwnersche Differentialgleichung und quasikonform fortsetzbare schlichte Funktionen," Journal für die reine und angewandte Mathematik, vol. 255, pp. 23-43, 1972.

[2] St. Ruscheweyh, "New criteria for univalent functions," Proceedings of the American Mathematical Society, vol. 49, no. 1, pp. 109-115, 1975. 
[3] Z. Nehari, Conformal Mapping, Dover, New York, NY, USA, 1975.

[4] P. Hamburg, P. Mocanu, and N. Negoescu, Analiză matematică (Funcţii complexe), Editura Didactică şi Pedagogică, Bucureşti, Romania, 1982.

[5] J. W. Alexander, "Functions which map the interior of the unit circle upon simple regions," Annals of Mathematics, vol. 17, no. 1, pp. 12-22, 1915.

[6] S. S. Miller, P. T. Mocanu, and M. O. Reade, "Starlike integral operators," Pacific Journal of Mathematics, vol. 79, no. 1, pp. 157-168, 1978.

[7] Y. J. Kim and E. P. Merkes, "On an integral of powers of a spirallike function," Kyungpook Mathematical Journal, vol. 12, pp. 249-252, 1972.

[8] N. N. Pascu and V. Pescar, "On the integral operators of Kim-Merkes and Pfaltzgraff," Mathematica, vol. 32(55), no. 2, pp. 185-192, 1990.

[9] D. Breaz and N. Breaz, "Two integral operators," Studia Universitatis Babeş-Bolyai. Mathematica, vol. 47, no. 3, pp. 13-19, 2002.

[10] G. I. Oros and G. Oros, "A convexity property for an integral operator $F_{m}$," in preparation.

[11] V. Pescar and S. Owa, "Sufficient conditions for univalence of certain integral operators," Indian Journal of Mathematics, vol. 42, no. 3, pp. 347-351, 2000.

[12] V. Pescar, "On some integral operations which preserve the univalence," The Punjab University. Journal of Mathematics, vol. 30, pp. 1-10, 1997. 